\title{
Perfil epidemiológico e análise microbiológica da infecção de sítio cirúrgico em pacientes humanos e animais de companhia ${ }^{1}$
}

\author{
Aline R. Murta ${ }^{2}$, Nilton B. Abreu Jr², Larissa S. Oliveira², Emily C. Carlo Reis², Fabrício \\ L. Valente ${ }^{2}$, Gustavo P. Gonçalves ${ }^{2}$, Renato B. Eleotério ${ }^{2}$ e Andréa P.B. Borges ${ }^{2 *}$
}

\begin{abstract}
Murta A.R., Abreu Jr N.B., Carlo Reis E.C., Valente F.L., Oliveira L.S., Gonçalves G.P., Eleotério R.B. \& Borges A.P.B. 2015. [Epidemiological profile and microbiological analysis of surgical site infection in human and pet patients.] Perfil epidemiológico e análise microbiológica da infecção de sítio cirúrgico em pacientes humanos e animais de companhia. Pesquisa Veterinária Brasileira 35(7):652-658. Departamento de Veterinária, Universidade Federal de Viçosa, Av. Peter Henry Rolfs s/n, Viçosa, MG 36570-900, Brazil. E-mail: andrea@ufv.br

Surgical site infection (SSI) has been indicated as the third cause of nosocomial infection. The present study aimed to determine the epidemiological profile of SSI and its association with the risk factors. It is a transversal study done at the São João Batista Hospital of Viçosa-MG and at the Surgery Service of the Small Animals Veterinary Hospital of the Universidade Federal de Viçosa-MG, from September 2012 to February 2013. Global SSI rates were $0.7 \%$ at the human and $3.46 \%$ at the veterinary hospitals. At the veterinary hospital, SSI rates were not related to contamination potential, with clean procedures presenting the greater rates. As for the type of surgery, orthopedic ones are the most common in both hospital and also the ones presenting the greater SSI rates. Surgeries during more than 120 minutes were $15.25 \%$ of the total of procedures at the human hospital and are even less common in the veterinary, with $1.26 \%$. Rate of SSI does not seem to be related to surgery duration in this classification. Bacteria isolated from surgical wounds were multi-resistant and the obtained data indicated that no criteria of antibiotic prophylaxis existed, mainly for clean surgeries. This scenario shows that the action of a commission to control nosocomial infection are extremely relevant in order to guarantee reliable data so that the quality of service may be evaluated and thus, promoting a decrease the risk of in post-operative complications.
\end{abstract}

INDEX TERMS: Hospital-acquired infection, infection control committee.

RESUMO.- A infecção de sítio cirúrgico (ISC) tem sido apontada como a terceira causa mais comum de infecção nosocomial. Este estudo objetivou determinar o perfil epidemiológico da ISC e sua associação aos fatores de risco descritos. Trata-se de um estudo transversal, realizado no Hospital São João Batista de Viçosa-MG e na Clínica Cirúrgica de Cães e Gatos do Hospital Veterinário da Universidade Federal de Viçosa-MG, no período de setembro de 2012 a fevereiro de 2013. As taxas globais de ISC foram de 0,7\%

\footnotetext{
${ }^{1}$ Recebido em 1 de abril de 2014.

Aceito para publicação em 15 de junho de 2015.

${ }^{2}$ Departamento de Veterinária, Universidade Federal de Viçosa (UFV), Av. P.H. Rolfs s/n, Viçosa, MG 36570-000, Brasil. *Autor para correspondência: andrea@ufv.br
}

no hospital humano e 3,46\% no veterinário. No hospital veterinário, a taxa de ISC não mostrou relação com o potencial de contaminação, apresentando a maior taxa nos procedimentos classificados como limpos. Quanto ao tipo de cirurgia, as ortopédicas são as mais comuns em ambos os hospitais e também as que apresentam maior taxa de ISC. Cirurgias com duração maior que 120 minutos corresponderam a $15,25 \%$ do total de procedimentos no hospital humano e são ainda menos comuns no veterinário, com 1,26\%. A taxa de ISC não parece estar relacionada à duração da cirurgia nesta estratificação. As bactérias isoladas das feridas cirúrgicas foram multirresistentes e os dados levantados indicam que não houve critério quanto ao emprego da antibioticoprofilaxia, principalmente nas cirurgias limpas. Este cenário mostra que é de extrema re- 
levância a atuação de uma comissão de controle de infecção hospitalar, a fim de garantir obtenção de dados fidedignos, para que se possa avaliar a qualidade do serviço prestado e assim promover a redução dos riscos de complicações pósoperatórias.

TERMOS DE INDEXAÇÃO: Infecção hospitalar, comissão de controle de infecção hospitalar.

\section{INTRODUÇÃO}

As infecções de sítio cirúrgico (ISC) são complicações pós-operatórias, que contribuem na elevação das taxas de morbimortalidade, custos hospitalares e prolongam o tempo de permanência dos pacientes nas instituições (Gaynes 2001). Em pacientes hospitalizados, as taxas de ISC correspondem a 14-16\% das infecções em serviços de saúde e ganham maior importância devido a fatores relacionados à população atendida e aos procedimentos realizados (Brasil 2009). Em medicina humana, assim como em medicina veterinária, os índices de infecção descritos variam bastante (Bernis Filho et al. 1998, Harari 2004). As Comissões de Controle de Infecção Hospitalar (CCIH) em medicina humana são obrigatórias nos hospitais do país (Brasil 1997), com diretrizes e normas bem estabelecidas (Brasil 1998). Porém, em Medicina Veterinária, não há controle dos dados estatísticos sobre a real incidência dessas infecções, devido à ausência das $\mathrm{CCIH}$, que são responsáveis pela vigilância epidemiológica de todos os pacientes, em especial aqueles que apresentam maior risco, como os cirúrgicos (Benedict et al. 2008, Weese 2008).

Segundo Akalin (2002), a antibioticoprofilaxia é considerada uma medida importante na prevenção das infecções. Entretanto, na atualidade, a resistência antimicrobiana constitui um crítico problema de saúde (Guardabassi et al. 2004, Mendes et al. 2005), uma vez que tem dificultado a seleção empírica dos antimicrobianos (Hoekstra \& Paultron 2002), pois cada vez mais os pacientes são tratados com antibióticos de amplo espectro, sem a certeza da sua real necessidade. Por isso, para evitar sua prescrição inadvertida e seu uso abusivo, o que favorece a seleção de cepas bacterianas resistentes, foram elaborados vários guias, com o objetivo de limitar a emergência de bactérias resistentes e reduzir a taxa de infecção das feridas cirúrgicas (ASHP 1999; Brasil 2001, Guilarde et al. 2009).

Dada à necessidade de maior conhecimento da ISC e dos fatores relacionados, o objetivo deste estudo foi determinar o perfil epidemiológico das infecções diagnosticadas, sua associação aos fatores de risco descritos, bem como o perfil de resistência antimicrobiana das culturas obtidas dos sítios de infecção em pacientes atendidos no Hospital São João Batista de Viçosa/MG e na Clínica Cirúrgica de Cães e Gatos do Hospital Veterinário da Universidade Federal de Viçosa/MG.

\section{MATERIAL E MÉTODOS}

O presente trabalho foi aprovado pelo Comitê de Ética no Uso de Animais e pelo Comitê de Ética em Pesquisa com Seres Humanos sob os protocolos números 47/2012 e 071010, respectivamente, da Universidade Federal de Viçosa.
A população foi constituída de pacientes humanos e animais de companhia admitidos no Hospital São João Batista de Viçosa-MG e na Clínica Cirúrgica de Cães e Gatos do Hospital Veterinário da Universidade Federal de Viçosa-MG, respectivamente, após serem submetidos a qualquer tratamento cirúrgico, no período de setembro de 2012 a fevereiro de 2013. Todos os procedimentos incluídos foram realizados na rotina de cada instituição, sem alterá-la. Os critérios de inclusão dos pacientes no estudo foram: (1) ter sido submetido à internação pré-cirúrgica, (2) ter sido levado ao bloco cirúrgico, (3) ter sofrido incisão em pele ou mucosa e a mesma ter sido fechada antes do paciente deixar o centro cirúrgico, (4) possuir prontuário preenchido, (5) não ser portador de infecção no ingresso no hospital e (6) ter comparecido ao retorno pós-operatório no ambulatório cirúrgico. Como critérios de exclusão foram considerados os casos em que os pacientes foram anestesiados para exame radiodiagnóstico e endoscópico, ocorrência de óbito durante a cirurgia e prontuários com ausência de informação ou apresentando incompreensão.

O diagnóstico da infecção de sítio cirúrgico ocorreu em até trinta dias após realização do procedimento. As respectivas instituições seguiram as recomendações da metodologia do National Healthcare Safety Network. Na clínica cirúrgica de cães e gatos, o diagnóstico ocorreu no ambulatório de retorno, as informações foram registradas em ficha específica, elaborada para este projeto e anexada ao prontuário e a análise das amostras foi realizada no Laboratório de Microbiologia da UFV. Já no Hospital São João Batista, os dados foram analisados através de um banco de dados fornecido pela própria instituição que já possui Comissão de Controle de Infecção Hospitalar. A taxa de infecção foi descrita para cada hospital segundo os parâmetros: (1) número total de procedimentos cirúrgicos; (2) sexo do paciente; (3) potencial de contaminação; (4) tipo de procedimento cirúrgico; (5) número de pessoas presentes na sala durante o procedimento; (5) duração do procedimento, maior ou menor que 120 minutos; (6) tempo até o diagnóstico da infecção. A taxa de ISC foi calculada para cada estrato dessas classificações.

Após o diagnóstico de infecção, realizou-se a higienização das margens das feridas cirúrgicas com solução salina 0,9\%. Foi colhido material da ferida cirúrgica com auxílio de um $s w a b$ estéril, inoculado em $1 \mathrm{ml}$ de $\mathrm{NaCl}$ 0,85\%, e estriado em ágar sangue de carneiro a $5 \%$ e ágar MacConkey, ambos incubados a $37^{\circ} \mathrm{C}$ por 24 horas. Transcorrido este tempo, as colônias isoladas obtidas em ambas as culturas que apresentaram morfologia macroscopicamente diferente, foram estriadas em ágar Brain-Heart-Infusion (BHI) para confirmação de culturas puras, em seguida, as culturas obtidas foram armazenadas em caldo BHI, adicionado $20 \%$ de glicerol e mantidas a $-80^{\circ} \mathrm{C}$. Após o término do período de colheita, os isolados que se encontravam armazenados foram reativados em ágar BHI para realização dos seguintes testes de triagem: coloração de Gram, provas de catalase e oxidase. As bactérias identificadas foram descritas segundo o tipo de procedimento cirúrgico realizado. Em ambos os hospitais, os antimicrobianos escolhidos para realização do antibiograma baseou-se na rotina cirúrgica de cada instituição e a classificação conforme coloração de Gram dos isolados obtidos.

\section{RESULTADOS}

No período de coleta dos dados, foram realizados 1579 procedimentos no hospital humano. Destes, 16 foram descartados do estudo por ausência de dados, totalizando assim 1563 amostras válidas. No hospital veterinário, dos 325 procedimentos totais, sete foram excluídos por ausência de dados ou óbito do paciente, totalizando 318 amostras 
válidas. Tanto no hospital humano quanto no veterinário, foram diagnosticados 11 casos de ISC, o que corresponde a uma taxa de ISC total de $0,70 \%$ e $3,46 \%$, respectivamente (Fig.1). Dos 318 pacientes atendidos no hospital veterinário, 32 eram felinos e nenhum deles apresentou ISC, mas foram contabilizados em todos os cálculos. No hospital humano, $52,97 \%$ dos pacientes eram do sexo masculino, que apresentou maior taxa de ISC que o feminino: respectivamente $1,08 \%$ e $0,27 \%$. No veterinário, $61,32 \%$ dos pacientes submetidos a algum procedimento cirúrgico no período avaliado eram fêmeas; entretanto, os machos apresentaram maior taxa de ISC: 4,88\% (Fig.2).

A maior parte dos procedimentos realizados em ambos os hospitais foi classificada como cirurgias limpas. No hospital humano, a frequência de cirurgias contaminadas somadas foi menor que 8\%. Esta foi uma diferença importante em relação ao hospital veterinário, onde, no período avaliado, as cirurgias contaminadas responderam por $18,24 \%$ e, juntamente com as infectadas, corresponderam a quase $25 \%$ das cirurgias realizadas. No hospital humano, houve ISC nos quatro níveis de classificação do potencial de contaminação e a taxa em que a ISC foi diagnosticada mostrou-se crescente da cirurgia limpa até a infectada. Já no hospital veterinário, a maior taxa de ISC foi encontrada naqueles procedimentos classificados como limpos, sendo que nenhum caso de infecção foi diagnosticado nos procedimentos potencialmente contaminados (Fig.3).

As cirurgias ortopédicas foram as mais comuns em ambos os hospitais: $31,99 \%$ no hospital humano e $24,21 \%$, no

\section{ISC Total}

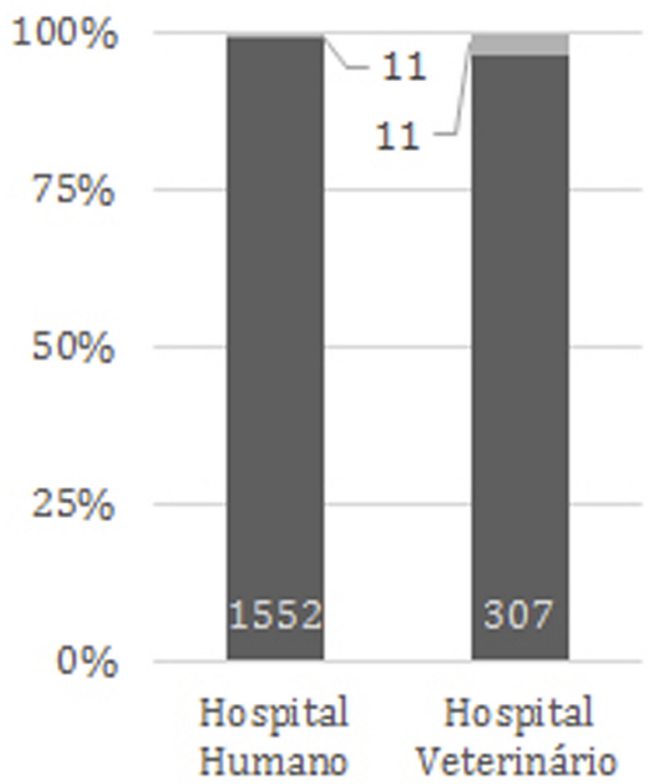

\section{ISC ausente $\quad$ ISC presente}

Fig.1. Taxa de infecção de sítio cirúrgico (ISC) diagnosticada nos procedimentos totais realizados em ambos os hospitais no período de seis meses. 0 número absoluto de casos está indicado nos gráficos.

\section{ISC segundo sexo}

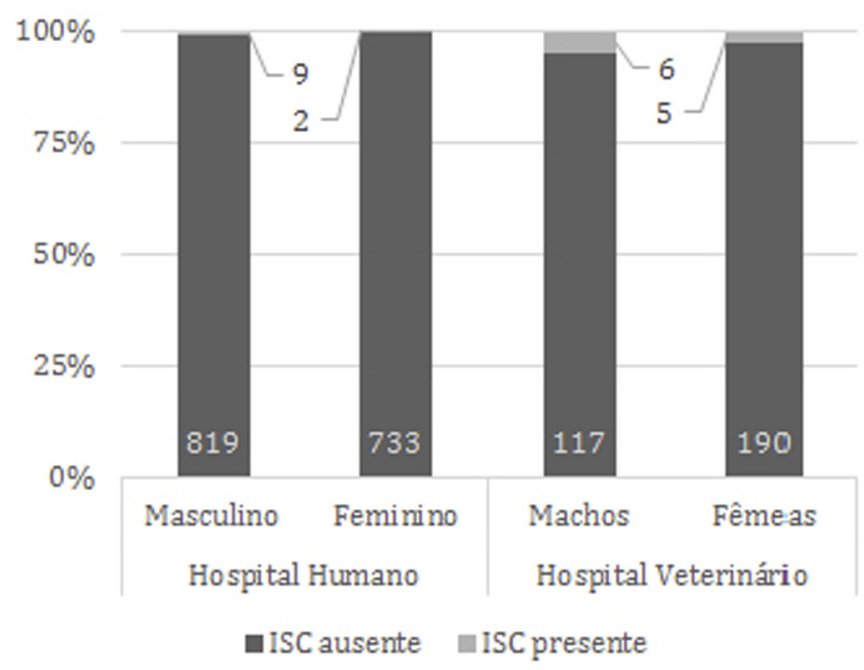

Fig.2. Taxa de infecção de sítio cirúrgico (ISC) diagnosticada nos procedimentos realizados em ambos os hospitais no período de seis meses, segundo o sexo dos pacientes. 0 número absoluto de casos está indicado nos gráficos.

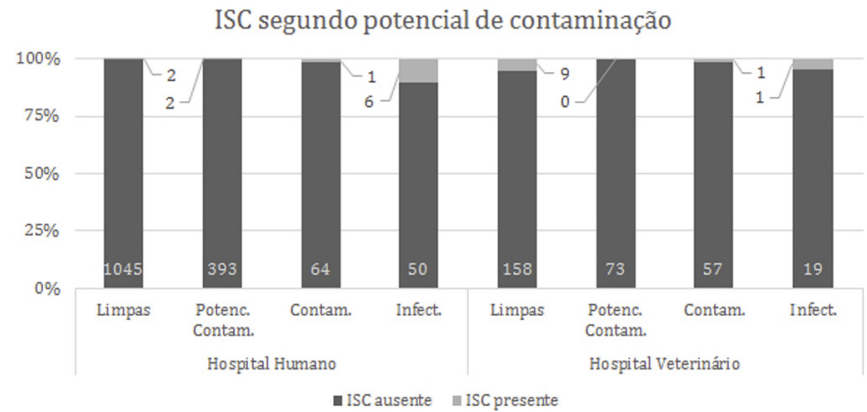

Fig.3. Taxa de infecção de sítio cirúrgico (ISC) diagnosticada nos procedimentos realizados em ambos os hospitais no período de seis meses, segundo o potencial de contaminação. 0 número absoluto de casos está indicado nos gráficos. Potenc. Contam.: potencialmente contaminadas; Contam.: contaminadas; Infec.: infectadas.

veterinário. No primeiro, elas são seguidas pelas cirurgias gerais, que correspondem a 23,03\%; enquanto no segundo, são seguidas pelas cirurgias ginecológicas, com 22,64\% dos procedimentos, e gerais, com $20,75 \%$. No hospital veterinário não ocorreram cirurgias estéticas no período estudado. Nem todos os tipos de procedimento apresentaram ISC. No hospital humano, as cirurgias ortopédicas, gerais, do sistema gastrointestinal e do sistema tegumentar apresentaram taxas específicas de ISC de 1,20\%, 0,56\%, 1,11\% e $0,98 \%$, respectivamente, sendo que esta correspondeu a apenas um caso diagnosticado. No hospital veterinário, 9,09\% das cirurgias ortopédicas, 8,33\% das cirurgias do sistema gastrointestinal (o que correspondeu a um caso) e 4,1\% das ginecológicas apresentaram ISC (Fig.4). Com a classificação das cirurgias em ortopédicas e não ortopédicas, estas tiveram taxa de ISC de $0,47 \%$ no hospital humano e 1,66\% no veterinário. 
ISC por tipo de cirurgia
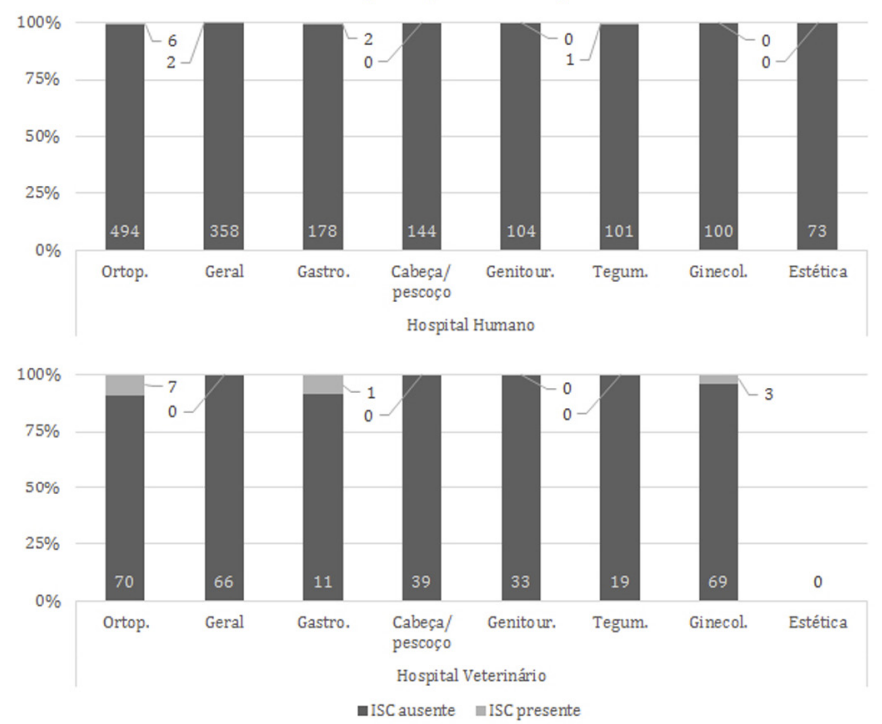

Fig.4. Taxa de infecção de sítio cirúrgico (ISC) diagnosticada nos procedimentos realizados em ambos os hospitais no período de seis meses, segundo o tipo de procedimento. 0 número absoluto de casos está indicado nos gráficos. Ortop.: ortopédico; Gastro.: gastrointestinal; Genitour: genitourinário; Tegum.: tegumentar; Ginecol.: ginecológico.

Cirurgias com duração maior que 120 minutos são mais comuns no hospital humano que no veterinário: $15,23 \%$ do total de procedimentos e $1,26 \%$, respectivamente. No veterinário, todos os casos de ISC diagnosticados foram em procedimentos que duraram menos de 120 minutos. No hospital humano, apenas um caso ocorreu em cirurgia mais longa que 120 minutos (Fig.5). Especificamente, o procedimento cirúrgico deste caso durou 4 horas e 15 minutos. No hospital humano, o diagnóstico de ISC ocorreu principalmente até o quinto dia após o procedimento, enquanto no veterinário entre 5 e 30 dias (Fig.6).

Foram identificados seis gêneros de bactérias nas infecções diagnosticadas no hospital humano, sendo 83,33\% caracterizados como Gram-negativos e 16,66\% Gram-positivos. A distribuição dos patógenos identificados é apresen- tada no Quadro 1. Em relação à resistência individual das bactérias aos antimicrobianos, a avaliação da ampicilina apresentou maior número de isolados resistentes entre as bactérias Gram-positivas, seguido por tetraciclina e amoxicilina/ácido clavulânico. Entre as bactérias Gram-negativas foi encontrada maior resistência antimicrobiana à tetraciclina, seguida por ampicilina, ciprofloxacina, amoxicilina/ ácido clavulânico, gentamicina e cefalotina. Pseudomonas

\section{ISC por duração de cirurgia}

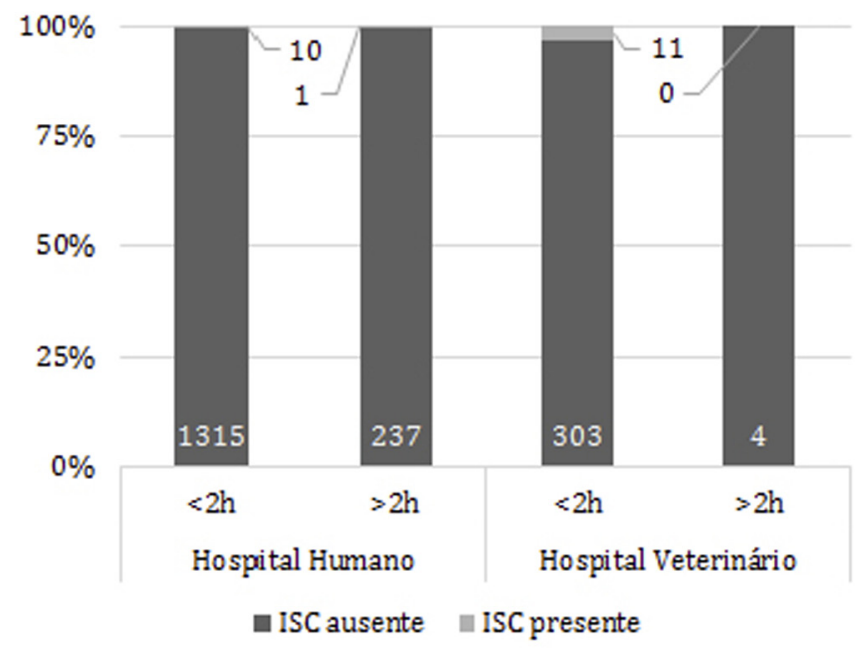

Fig.5. Taxa de infecção de sítio cirúrgico (ISC) diagnosticada nos procedimentos realizados em ambos os hospitais no período de seis meses, segundo a duração do procedimento. 0 número absoluto de casos está indicado nos gráficos.

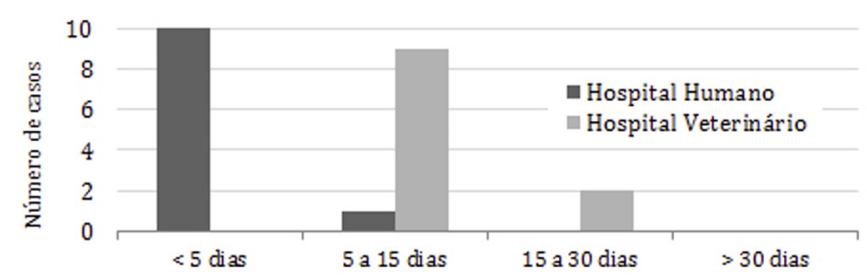

Fig.6. Distribuição dos casos de infecção de sítio cirúrgico (ISC) segundo o tempo para o diagnóstico.

Quadro 1. Distribuição dos gêneros de microrganismos nos casos de infecção de sítio cirúrgico identificados nos Hospitais São João Batista e

Hospital Veterinário da Universidade Federal de Viçosa, ambos em Viçosa-MG, no período de setembro de 2012 a fevereiro de 2013

\begin{tabular}{|c|c|c|c|c|}
\hline \multirow[t]{2}{*}{ Gênero / Espécie } & \multicolumn{2}{|c|}{ Hospital São João Batista } & \multicolumn{2}{|c|}{ Hospital Veterinário } \\
\hline & $\begin{array}{l}\text { Número de } \\
\text { isolados }\end{array}$ & $\begin{array}{c}\text { Resistência a } \\
\text { antimicrobianos }\end{array}$ & $\begin{array}{l}\text { Número } \\
\text { de isolados }\end{array}$ & $\begin{array}{c}\text { Resistência a } \\
\text { antimicrobianos }\end{array}$ \\
\hline \multicolumn{5}{|l|}{ Gram-negativo } \\
\hline Citrobacter & 1 & $\mathrm{~b}, \mathrm{c}, \mathrm{g}$ & 0 & \\
\hline Enterobacter sp. & 3 & $a, b, e, g$ & 0 & \\
\hline Escherichia coli & 3 & $\mathrm{e}, \mathrm{f}, \mathrm{g}$ & 0 & \\
\hline Klebsiella sp. & 2 & $b, e, g$ & 0 & \\
\hline Pseudomonas sp. & 1 & $\mathrm{e}$ & 0 & \\
\hline \multicolumn{5}{|l|}{ Gram-positivo } \\
\hline Staphylococcus coagulase - & 1 & $\mathrm{a}, \mathrm{b}$ & 7 & $b, c, d, e, f, g$ \\
\hline Staphylococcus aureus & 1 & & 4 & \\
\hline
\end{tabular}

a: Amoxicilina/Ácido clavulânico; b: Ampicilina; c: Cefaloxina; d: Cefalotina; e: Ciprofloxacina; f: Gentamicina; g: Tetraciclina. 
sp. foi resistente somente à ciprofloxacina. No hospital veterinário foram isoladas apenas bactérias Gram-positivas do gênero Staphylococcus, que apresentou resistência a quase todos os antibióticos testados. É importante salientar que em todas as feridas cirúrgicas classificadas como limpas os pacientes humanos e animais receberam antibioticoprofilaxia no pré-operatório e o diagnóstico das infecções ocorreu em até 30 dias após a cirurgia. Ao observar o perfil de resistência múltipla dos microrganismos, verificou-se que praticamente todos os isolados foram multirresistentes, sendo que a maioria apresentou resistência para mais de um grupo de antimicrobianos, chegando a seis deles.

\section{DISCUSSÃO}

A importância do controle da ISC é reconhecida na medicina humana e sua relevância na veterinária também aponta para a necessidade de uma metodologia mais criteriosa de controle (Braga 2008, Corsini 2012). As diferenças e a diversidade entre instituições, seus procedimentos e sua população atendida poderiam ser fatores limitantes na criação de diretrizes, porém, mesmo para a medicina humana, essas questões são consideradas (Brasil 2009). 0 Hospital São João Batista de Viçosa conta com a atuação de uma CCIH, porém no Hospital Veterinário da Universidade Federal de Viçosa não existe a formalização desse controle e, em ambas as instituições não se realiza o seguimento do paciente cirúrgico após sua alta hospitalar, o que é considerado de extrema importância nos pacientes que apresentam risco para infecção, em especial os cirúrgicos, pois as taxas de infeção podem ser subnotificadas (Ferraz et al. 1995, Oliveira \& Carvalho 2004, Macbeth et al. 2005, Starling 2011). Por isso, torna-se imprescindível que cada instituição adote o melhor método de seguimento do paciente, que se enquadra à sua realidade, seja por contato telefônico, carta resposta, busca ativa, ambulatório de egresso, ou outro método eficaz (Gravel-Tropper et al. 1995), pois a inexistência de um programa de vigilância epidemiológica das infecções pode ser considerada um importante fator de risco no ambiente hospitalar (Couto \& Pedrosa 2003).

Em estudos realizados no mesmo hospital veterinário, Braga (2008) e Corsini (2012) verificaram taxas globais de ISC de $9,5 \%$ e $5,25 \%$, respectivamente. Um dos fatores que podem explicar as diferentes taxas encontradas é a alteração das equipes responsáveis pela rotina na Clínica Cirúrgica de Cães e Gatos do Hospital Veterinário da Universidade Federal de Viçosa, visto que ela é formada não apenas por professores e técnicos, mas também por residentes, que não fazem parte do quadro permanente. Embora haja algumas diferenças nas metodologias desse e dos dois trabalhos anteriores, é possível que o tratamento dado ao assunto dentro do hospital tenha contribuído para a diminuição da taxa global de ISC para os 3,46\% encontrados neste trabalho. Este valor também está abaixo dos encontrados em dois outros hospitais veterinários universitários: 7,96\% e 8,5\%, descritos, respectivamente por Arias et al. (2013) e Rodrigues (2013).

A alta taxa de ISC detectada nas cirurgias limpas realizadas no hospital veterinário sugere haver algum outro fator de risco não relacionado ao potencial de contaminação da ferida cirúrgica. Por isso, deve-se levar em consideração o fato de este estudo ter sido realizado em um hospital escola, onde há grande fluxo de pessoas, bem como os riscos relacionados aos pacientes ou cirurgiões. Estes resultados indicam um ponto que necessita intervenção mais aprofundada nessa instituição e que afetará positivamente as taxas globais de ISC. Para alguns autores, é insuficiente utilizar somente a correlação da classificação da ferida cirúrgica para avaliar o potencial risco de desenvolver infecção, uma vez que somente essa classificação não leva em consideração o risco do paciente, além de existirem variações nas taxas entre diferentes tipos de procedimentos que apresentam a mesma classificação (Vasseur et al. 1988, Brown et al. 1997, Eugster et al. 2004).

Nos dois hospitais houve maior prevalência de ISC nas cirurgias ortopédicas. De fato, este tipo de procedimento é considerado o mais suscetível à infecção (Dale et al. 2011), constituindo fator de risco, pois tem a capacidade de promover colonização bacteriana, levando à formação de um biofilme, que dificulta uma resposta satisfatória do sistema imunológico (Smith et al. 1989, Khan et al. 2008). Os estudos anteriores realizados no hospital veterinário da Universidade Federal de Viçosa também apontaram as cirurgias ortopédicas como os procedimentos que se apresentaram em maior quantidade, conforme os tipos de procedimentos realizados, identificando taxas de ISC que variaram de 14,4\% e 8,65\% (Braga 2008, Corsini 2012).

A duração da cirurgia está diretamente relacionada à ocorrência de ISC, pois quanto maior for a duração do procedimento, maior é a exposição dos tecidos e a suscetibilidade da equipe à fadiga, o que pode levar a falhas técnicas (Cruse \& Foord 1980, Garibaldi et al. 1991, Ehrenkranz et al. 1995, Mangram et al. 1999). No presente estudo, as cirurgias com duração maior que 120 minutos foram pouco comuns, principalmente no hospital veterinário e a taxa de ISC não foi mais frequente nas cirurgias mais longas. No hospital humano, os pacientes tiveram o diagnóstico de ISC durante sua internação. Já no veterinário, os animais foram diagnosticados entre 5 e 15 dias, no retorno ambulatorial, quando eram trazidos pelos proprietários para retirada de pontos cirúrgicos, consulta ou realização de exames. Conforme Mangram et al. (1999) e Oliveira \& Ciosak (2004) as ISC podem ser diagnosticadas em até 30 dias após o procedimento, ou até um ano, em caso de colocação de prótese metálica.

Os resultados encontrados nos hospitais sugerem utilização inadequada da antibioticoprofilaxia em todas as cirurgias limpas, que não se enquadraram nos casos especiais, em que é indicada, divergindo das recomendações de Daschner (1989) e Condon \& Wittmann (1991) os quais relatam que a utilização de antibioticoprofilaxia em cirurgias limpas não diminui a taxa de infecção da ferida cirúrgica. Por isso, nestes tipos de cirurgia os benefícios não se sobrepõem aos riscos, de modo que não se recomenda sua utilização (Baum et al. 1981, Platt et al. 1990), exceto em casos especiais, como cirurgias com duração maior que 90 minutos, utilização de próteses e neurocirurgias, pacientes acima de 70 anos, desnutridos e imunodeprimidos (Dellinger 2003).

Nos hospitais, os microrganismos isolados nas feridas cirúrgicas pertencentes ao grupo das bactérias Gram-posi- 
tivas foram Staphylococcus sp. Santos et al. (1997) realizaram estudo em pacientes humanos e concluíram que as ISC pode ser provocadas por diversos microrganismos, sendo frequentemente causadas por bactérias colonizantes da pele do próprio paciente, sendo identificado Staphylococcus em $33,9 \%$ dos casos. Porém, em outro estudo, conduzido por Naylor et al. (2001), Staphylococcus foi isolado com maior frequência em cirurgias com menor grau de contaminação, como as limpas.

Esses dados foram condizentes com aqueles encontrados no hospital veterinário, uma vez que somente bactérias Gram-positivas do gênero Staphylococcus foram isoladas nas feridas cirúrgicas limpas. Garibaldi et al. (1991) acompanharam ISC em pacientes humanos durante quatro anos, identificando em cultura de colheita intra-operatória os microrganismos Staphylococcus (78\%) e Escherichia (11\%). Já Abdel-Fattah (2005) isolou Pseudomonas, Klebsiella, Citrobacter e Enterobacter, identificando maior taxa de bactérias Gram-negativas em comparação às Gram-positivas, concordando com os dados encontrados no hospital humano. Já Ishii et al. (2011) ao realizarem estudo em hospital veterinário, detectaram maiores taxas de bactérias Gram-positivas em comparação às Gram-negativas, concordando com os dados encontrados no hospital veterinário. Porém, estes dados diferem daqueles obtidos por Corsini (2012) que identificou 12 gêneros de microrganismos, dentre eles $62,93 \%$ caracterizados como Gram-negativos e $36,07 \%$ Gram-positivos. Meyers et al. (2007) encontraram alta resistência antimicrobiana à amoxicilina associada ao ácido clavulânico e às cefalexinas.

Oliveira et al. (2005) encontraram um perfil elevado de resistência à tetraciclina, concordando com os resultados encontrados no hospital humano, no qual todas as bactérias isoladas foram resistentes à tetraciclina, com exceção de Pseudomonas. Lichtenfels et al. (2007) obtiveram alta resistência a ampicilina, ciprofloxacina, clindamicina e gentamicina. No hospital veterinário, a bactéria Staphylococcus apresentou resistência a todos os antimicrobianos testados, exceto a amoxacilina/ácido clavulânico. As ISC e a resistência bacteriana podem causar grande impacto no futuro da Medicina Humana e Veterinária, prejudicando os tratamentos propostos, além de elevar os custos dos tratamentos, com perda do acesso ao antibiótico efetivo (Aiello et al. 2007). Por isso, com o aumento do número de bactérias multirresistentes torna-se necessário o desenvolvimento e a implementação de medidas para monitorar e controlar a difusão de resistência, que é um problema bastante atual e muito prevalente em pacientes cirúrgicos (Umber \& Bender 2009).

Assim, reforça-se a relevância de um serviço de vigilância epidemiológica dentro das instituições, bem como o seguimento pós-alta dos pacientes cirúrgicos, a fim de garantir a obtenção de dados fidedignos, para que se possa avaliar a qualidade do serviço prestado e, assim, promover a redução dos riscos de complicações pós-operatórias. Além disso, a utilização de critérios para antibioticoprofilaxia e o monitoramento constante do perfil de resistência bacteriana devem ser estabelecidos. Embora os hospitais veterinários apresentem diferenças importantes em rela- ção aos humanos, muitos aspectos comuns devem ser observados para o desenvolvimento das práticas hospitalares e da promoção da saúde.

\section{REFERÊNCIAS}

Abdel-Fattah M. 2005. Surveillance of nosocomial infections at a Saudi Arabian military hospital for one-year period. Ger. Med. Sci. 6:01-10.

Aiello G., Battaglia L., Arias M.V. \& Freitas J.C. 2007. Determinação dos índices de infecção hospitalar em um centro cirúrgico universitário veterinário de pequenos animais. Acta Scient. Vet. 35:345-346.

Akalin H. 2002. Surgical prophylaxis: the evolution of guidelines in an area of cost containment. J. Hosp. Infect. 50:03-07.

ASHP (American Society of Health-System Pharmacists) 1999. Therapeutic Guidelines on Antimicrobial Prophylaxis in Surgery. Am. J. Health Syst. Pharm. 56:1839-1888.

Arias M.V.B., Aiello G., Battaglia L.A. \& Freitas J.C. 2013. Estudo da ocorrência de infecção hospital em cães e gatos em um centro cirúrgico veterinário universitário. Pesq. Vet. Bras. 33(6):771:779.

Baum M.L., Anish D.S., Chalmers T.C., Sacks H.S., Smith Jr H. \& Fagerstrom R.M. 1981. A survey of clinical trials of antibiotic prophylaxis in colon surgery: evidence against further of no treatment controls. N. Engl. J. Med. 14:795-799.

Benedict K.M., Morley P.S. \& Van Metre D.C. 2008. Characteristics of biosecurity and infection control programs at veterinary teaching hospitals. J. Am. Vet. Med. Assoc. 5:767-773.

Bernis Filho W.O., Rezende C.M.F., Abreu V.L.V. \& Bernis V.M.O. 1998. Infecções hospitalares em feridas cirúrgicas de pequenos animais. Arq. Bras. Med. Vet. Zootec. 50(2):127-132.

Braga D.P. 2008. Incidência e fatores de risco associados à infecção do sítio cirúrgico na clínica de cães e gatos do Hospital Veterinário da Universidade Federal de Viçosa. Dissertação de Mestrado em Medicina Veterinária, Universidade Federal de Viçosa, MG. 104p.

Brasil 1997. Lei no 9.431, de 6 de janeiro de 1997. Dispõe sobre a obrigatoriedade da manutenção de programa de controle de infecções hospitalares pelos hospitais do País. Disponível em <https://www.planalto.gov. br/ccivil_03/leis/19431.htm> Acesso em 14 out. 2013.

Brasil 1998. Ministério da Saúde. Portaria no 2616, de 12 de maio de 1998. Expedir diretrizes e normas para prevenção e o controle das infecções hospitalares. Disponível em <http://bvsms.saude.gov.br/bvs/saudelegis/gm/1998/prt2616_12_05_1998.html> Acesso em 14 out. 2013.

Brasil 2001. Consenso sobre o uso racional de antimicrobianos. Agência Nacional de Vigilância Sanitária, Ministério da Saúde, Brasília, DF. 36p.

Brasil 2009. Sítio Cirúrgico: critérios nacionais de infecção relacionada à assistência à saúde. Agência Nacional de Vigilância Sanitária, Ministério da Saúde, Brasília, DF. 19p.

Brown D.C., Conzemius M.G., Shofer F. \& Swann H. 1997. Epidemiologic evaluation of post-operative wound infection in dogs and cats. J. Am. Vet. Med. Assoc. 9:1302-1306.

Condon R.E. \& Wittmann D.H. 1991. The use of antibiotics in general surgery. Curr. Prob. Surg. 12:807-907.

Corsini C.M. 2012. Avaliação da incidência de infecção do sítio cirúrgico, em pacientes e gatos operados no hospital veterinário da UFV, associados a fatores de risco, bactérias isoladas e perfil de resistência aos antimicrobianos. Dissertação de Mestrado em Medicina Veterinária, Universidade Federal de Viçosa, Viçosa, MG. 69p.

Couto R. \& Pedrosa T. 2003. Infecção Hospitalar e outras Complicações não infecciosas da doença-epidemiologia, Controle e Tratamento. $3^{\underline{a}}$ ed. Medsi, Rio de Janeiro. 904p.

Cruse P. \& Foord R. 1980. The epidemiology of wound infection: a 10-yearprospective study of 62,939 wounds. Surg. Clin. North. Am. 60:27-40.

Dale H., Skramm I., Løwer H.L., Eriksen H.M., Espehaug B., Furnes O., Skjeldestad F.E., Havelin L.I. \& Engesaeter L.B. 2011. Infection after primary hip artroplasty: a comparison of 3 Norwegian registers. Acta Orthop. 82:646-654. 
Daschner F.D. 1989. Who Symposium: use and abuse of antibiotics worldwide. Infection 17:46-57.

Dellinger E. 2003. Infecções cirúrgicas e escolha dos antimicrobianos, p.182-200. In: Townsend C. (Ed.), Sabiston Tratado de Cirurgia. 6 $6^{\underline{a}}$ ed. Guanabara Koogan, Rio de Janeiro.

Ehrenkranz N.J., Richter E.I., Phillips P.M. \& Shultz J.M. 1995. An apparent excess of operative site infections: Analysis to evaluate false-positive diagnoses. Infect. Control. Hosp. Epidemiol. 16:712-716.

Eugster S., Schawalder P., Gaschen F. \& Boerlin P. 2004. A prospective study of postoperative surgical site infections in dogs and cats. Vet. Surg. 33:542-550

Ferraz E.M., Ferraz A.A., Coelho H.S., Pereira Viana V.P., Sobral S.M., Vasconcelos M.D. \& Bacelar T.S. 1995. Postdischarge surveillance for nosocomial wound infections: does judicious monitoring find cares? Am. J. Infect. Control 23:290-294.

Garibaldi R.A., Cushing D. \& Lerer T. 1991. Risk factors for postoperative infection. Am. J. Med. 91:158-163.

Gaynes R. 2001. Surgical-site infections (SSI) and the NNIS basic SSI risk index, part II: room for improvement. Infect. Control Hosp. Epidemiol. 22:266-267.

Gravel-Tropper D., Oxley C., Memish Z. \& Garber G.E. 1995. Underestimation of surgical site infection rates in obstetrics and gynecology. Am. J. Infect. Control 23:22-26.

Guardabassi L., Schwarz S. \& Lloyd D.H. 2004. Pet animals as reservoirs of antimicrobial resistant bacteria. J. Antimicrob. Chemother. 54:321-332.

Guilarde A., Pacheco I.T., Gomes L.V., Lima V.A., Pacheco J.P. \& Sousa M.A. 2009. Avaliação da antibioticoprofilaxia cirúrgica em hospital universitário. Revta Patol. Trop. 38:179-185.

Harari J. 2004. Controle de infecção, p.25-32. In: Harari J. (Ed.), Segredos em Cirurgias de Pequenos Animais. Artmed, São Paulo.

Hoekstra K. \& Paultron R. 2002. Clinical prevalence and antimicrobial susceptibility of Staphylococcus aureus and Staphylococcus intermedius in dogs. J. Appl. Microbiol. 93:406-413.

Ishii J.B., Freitas J.C. \& Arias M.V.B. 2011. Resistência de bactérias isoladas de cães e gatos no Hospital Veterinário da Universidade Estadual de Londrina. Pesq. Vet. Bras. 31:533-537.

Khan M.S., ur Rehman S., Ali M.A., Sultan B. \& Sultan S. 2008. Infection in orthopedic implant surgery, its risk factors and outcome. J Ayub. Med. Coll. Abbottabad. 20:23-25.

Lichtenfels E., Lucas M.L., Webster R. \& D’Azevedo P.A. 2007. Profilaxia antimicrobiana em cirurgia vascular periférica: a cefazolina ainda é o padrão-ouro? J. Vasc. Bras. 06:378-387.

Macbeth D., Gardner G., Wallis M. \& Gerrard J. 2005. Surgeons perspectives on surgical wound infections rate date in Queensland. Am. J. Infect. Control 33:97-103.
Mangram A.J., Horan T.C., Pearson M.L., Silver L.C. \& Jarvis W.R. 1999. Guideline for prevention of surgical site infection. Hospital Infection Control Practices Advisory Committee. Infect. Control Hosp. Epidemiol. 20:250-278

Mendes C., Oplustil C., Sakagami E., Turner P. \& Kiffer C. 2005. Antimicrobial susceptibility in Intensive Care Units: MYSTIC Program Brazil 2002. Braz. J. Infect. Dis. 09:44-51.

Meyers B., Schoeman J.P., Goddard A. \& Picard J. 2007. The bacteriology and antimicrobial susceptibility of infected and non-infected dog bite wounds: Fifty cases. Vet. Microbiol. 127:360-368.

Naylor A.R., Hayes P.D. \& Darke S. 2001. A Prospective Audit of Complex Wound and Graft Infection in Great Britain and Ireland: Emergence of MRSA. Eur. J. Vasc. Endovasc. Surg. 21:289-294.

Oliveira A. \& Carvalho D. 2004. Postdischarge surveillance: The impact on surgical site infection incidence in a Brazilian university hospital. Am. J. Infect. Control 32:358-361.

Oliveira A. \& Ciosak S. 2004. Infecção do sítio cirúrgico no segmento pósalta: impacto na incidência e avaliação dos métodos utilizados. Revta. Esc. Enferm. USP 38:379-385.

Oliveira L.C., Medeiros C.M.O., Silva I.N.G., Monteiro A.J., Leite C.A.L. \& Carvalho C.B.M. 2005. Susceptibilidade a antimicrobianos de bactérias isoladas de otite externa em cães. Arq. Bras. Med. Vet. Zootec. 57:405-408.

Platt R., Zaleznik D.F., Hopkins C.C., Dellinger E.P., Karchmer A.W., Bryan C.S., Burke J.F., Wikler M.A., Marino S.K., Holbrook K.F., Tosteson T.D. \& Segal M.R. 1990. Perioperative antibiotic prophylaxis for herniorrhaphy and breast surgery. N. Engl. J. Med. 322:153-160.

Rodrigues E.M.P. 2013. Infecção de sítio cirúrgico em cães e gatos na rotina do bloco cirúrgico de hospital veterinário universitário em Porto Alegre, no ano de 2012. Dissertação de Mestrado em Ciências Veterinárias, Universidade Federal do Rio Grande do Sul, Porto Alegre, RS. 94p.

Santos K.R., Fonseca L.S., Bravo Neto G.P. \& Gontijo Filho P.P. 1997. Surgical Site Infection: rates, etiology and resistence patterns to antimicrobials among strains isolated at Rio de Janeiro University Hospital. Am. J. Infection. Control 25:217-220.

Smith M.M., Vasseur P.B. \& Saunders H.M. 1989. Bacterial growth associated with metallic implants in dogs. J. Am. Vet. Med. Assoc. 195:765-767.

Starling C. 2011. Surgical site infection in patients submitted to orthopedic surgery: the NNIS risk index and risk prediction. Revta Lat.-Am. Enferm. 19:269-276.

Umber J.K. \& Bender J.B. 2009. Pets and antimicrobial resistance. Vet. Clin. North. Am., Small Anim. Pract. 39:279-292.

Vasseur P.B., Levy J., Dowd E. \& Eliot J. 1988. Surgical wound infection rates in dogs and cats. Vet. Surg. 17:60-64.

Weese J. 2008. A review of post-operative infections in veterinary orthopaedic surgery. Vet. Comp. Orthop. Traumatol. 21:99-105. 